\title{
VARIA
}

\section{LOS PLATEROS DE CUENCA EN EL ÚLTIMO CUARTO DEL SIGLO XVIII Y SUS RELACIONES PROFESIONALES CON EL AYUNTAMIENTO DE LA CIUDAD}

La ciudad de Cuenca tuvo su momento de esplendor en el siglo xvi. A ello contribuyó, en buena medida, la pujanza económica de la provincia gracias a su ganado lanar en el medio rural, y a las industriales textiles y las derivadas de éstas en la capital, así como el buen nivel cultural de sus clases dominantes, fundamentalmente del alto clero. Ambas circunstancias favorecieron la producción artística, sobre todo la de la platería que alcanzó cotas muy altas. Se sucedieron varias generaciones de extraordinarios plateros encabezados por Francisco Becerril, creador y magnífico artista, que convirtió a este centro platero en difusor de tipologías y motivos ornamentales ${ }^{1}$.

Pero ya en los últimos años del siglo se puede apreciar el inicio de una crisis económica que alcanzó tintes de auténtica catástrofe en el siglo XVII ${ }^{2}$. A la crisis económica siguió la despoblación de la ciudad que quedó reducida a la cuarta parte. El siglo xviII verá una incipiente recuperación, sostenida a lo largo de todo el siglo, salvo un paréntesis en los años centrales que dio lugar a la revuelta de 1766, común, por otro lado, a varios puntos de la geografía española, pero que no consiguió hacerla remontar el vuelo totalmente.

El estudio de la plata conquense en los siglos XVII y xvIII, salvo algunos trabajos parciales ${ }^{3}$, está aun por hacer, por lo que con este artículo, elaborado con los datos documentales obtenidos en el Archivo Municipal de Cuenca, quiero iniciar una aproximación al tema, aportando una visión de todo lo relativo a la relación de los plateros con el Ayuntamiento en el último cuarto del s. XVIII, atendiendo muy especialmente a la manera en que les afectaron los profundos cambios que transformaron al gremio.

En primer lugar, podemos observar una reducción drástica en el número de plateros que trabajaban en Cuenca. Se puede comprobar a través de los censos que se hicieron por diversos motivos, generalmente con fines económicos, para las quintas de soldados o para el reparto de los que vienen de fuera, ya que, aunque la mayoría están muy incompletos, son bastante signi-

\footnotetext{
${ }^{1}$ A. López-Yarto: Francisco Becerril, Madrid, 1991; idem: La orfebrería del siglo xvı en la provincia de Cuenca, Cuenca, 1998.

${ }^{2}$ La génesis de la decadencia económica de Cuenca está magníficamente estudiada por M. Jiménez Monteserín: Vere pater pauperum: el culto a San Julián en Cuenca, Cuenca, 1999.

${ }^{3}$ F.A. Martín: «El punzón de Cuenca», en Goya, no 151, 1979, pp. 12-18. A. López-Yarto: «Pervivencia de modelos renacentistas en la obra de un platero conquense del siglo xvir: Juan de Castilla», en Velázquez y el Arte de su tiempo. IV Jornadas de Arte, Madrid, 1991, pp. 365-372; idem: «Un manuscrito del platero Juan de Castilla referente a la custodia de la catedral de Cuenca, obra de Francisco Becerril», en Estudios de Platería. San Eloy 2003, Murcia, 2003, pp. 313-327.
} 
ficativos. Según el Catastro del Marqués de la Ensenada, que en Cuenca se hizo en 1752, en ese año había en Cuenca 1.692 vecinos y 1.250 casas entre las que se incluyen las arruinadas. En él figuran, entre los oficios, seis maestros plateros, un oficial, un anexo y cuatro aprendices. Según el propio documento «intitulan así [un anexo] por no ser todavía oficial». Los maestros en esos momentos ganan en torno a los 5 y 8 reales al día, que es la misma cantidad que cobran los arquitectos, maestros albañiles, doradores y pintores. Cada oficial gana 4 reales, el anexo 3 y los aprendices real y medio. Entre todos ellos destaca Antonio Yanguas que era el encargado del mantenimiento del reloj de la ciudad, por lo que cobraba 618 reales al año ${ }^{4}$.

Conocemos ciento setenta y cinco nombres de plateros del s. XvI, cuarenta y ocho trabajan en el primer tercio, sesenta y uno en el segundo y sesenta y seis en el tercero. Sin embargo en el XVIII hay una media de seis en cada censo, aunque en el de.1772 llega a haber nueve. Como ejemplo de lo que pasaba en otras ciudades, en 1771 se reúnen los agremiados de Valladolid para comentar las nuevas ordenanzas y son veintitrés plateros ${ }^{5}$. Igual que en el XVI, hay diversos miembros de una misma familia dedicados al Arte de la plata, aunque es difícil poder decir con seguridad el grado de parentesco que hay entre ellos. Así, el ya citado Antonio Yanguas, que, además de cuidar el reloj de la ciudad era platero de la catedral, en el censo de 1771 tiene cuarenta y siete años, puede ser el padre de Alejandro Yanguas que en 1800 tenía treinta y nueve. Francisco Casado, que en 1771 tenía treinta y nueve años, puede ser el padre de Julián Casado que figura en el censo de 1805 como platero. Y, por último, Juan Serrano Briones, quizá el más sobresaliente de todos, que en 1771 tiene treinta y seis años, es el padre de Jacinto Serrano, tal y como consta en el de 1800 en el que éste tiene veinticuatro años.

En el siglo XVIII, los plateros de Cuenca siguen viviendo, en su mayoría, en torno a las parroquias de Santa Cruz y Santiago, situada esta última en la Catedral, lo mismo que en el xvi. Pero hay algunos que se apartan de estos barrios y fijan su residencia en las parroquias de Santa María de Gracia, en la antigua judería, San Pedro, San Gil o San Juan ${ }^{6}$. En torno a 1812 la dispersión es mayor, y a las parroquias antes citadas hay que añadir las de San Andrés y San Esteban.

También podemos observar que no hay movilidad entre los barrios. Es muy posible que se cambiaran de casa varias veces, como lo hacían otros artistas, cosa que había sido muy frecuente en el siglo XVI, pero siempre escogen la nueva vivienda en torno a la misma parroquia ${ }^{7}$.

En cuanto a la importancia socioeconómica que llegaron a tener, no contamos con más datos que los aportados por el Catastro del Marqués de la Ensenada, recogidos en párrafos anteriores, los cuales nos muestran que los plateros venían a ganar lo mismo que los maestros de otras manifestaciones artísticas. Más adelante veremos como Juan Serrano Briones, que era de los que más trabajo tenían, no puede pagarse el viaje a Madrid para examinarse ante la Junta de Comercio. Esto nos indica que, basándonos en que en el censo pone que sólo cobraban el día que trabajaban, es muy posible que muchos días no tuvieran trabajo.

Desde hace tiempo sabemos la importancia que tuvieron los Gremios y Hermandades en el desarrollo de la actividad profesional y privada de los artistas desde la Edad Media, y de forma muy especial en la de los plateros por el control que sufrían al utilizar un material muy

\footnotetext{
${ }^{4}$ AMC. Legajo 135, expediente 1, fols. 222, 286 y 286v.

${ }^{5}$ C. Heredia: «El platero vallisoletano Hipólito Bercial del Valle y la Junta de Comercio y Moneda de Madrid», en III Jornadas de Arte. Cinco siglos de Arte en Madrid (XV-XX), Madrid, 1991, p. 352.

${ }^{6}$ Tomás López, Juan Serrano Briones, Antonio Yanguas, Ignacio de Andrés, Mariano Aparicio y Juan Lorenzo en Santa Cruz; Juan de la Fuente, Francisco Casado y Juan Pérez en la de Santiago; Manuel Casado y Juan de Ayllón en la de Santa María de Gracia; Juan Antonio Aguilar en San Pedro y Julián Casado en San Gil, aunque a veces figura en la de San Juan.

${ }^{7}$ Los censos figuran en el AMC. Legajo 904, expedientes 3, 4, 6. Legajo 905, expedientes 1, 2, 4, 5. Legajo 906, expedientes $1,4,5$.
}

$A E A$, LXXVII, 2004, 306, pp. 171 a 201 
caro. Curiosamente en Cuenca parece que, durante mucho tiempo, no hubo ni lo uno ni lo otro. Esto se deduce de la documentación conservada. En el s. XVI, en el momento de mayor esplendor y de mayor número de artistas en activo, sólo tenemos una noticia acerca del tema y bastante incompleta. En 1519 el peostre y cofrades de los «plateros, herreros y oficiales de martillo» piden al Ayuntamiento la aprobación de unas ordenanzas que han redactado reunidos en cabildo. La solicitud, que está sin firmar, es presentada en la junta del 6 de diciembre de 1519, encargando la corporación a Fernando Valdés que las estudie y presente un informe. Pero en los libros de actas del Concejo no vuelve a haber ninguna noticia más. Los plateros, según se deduce de la solicitud, rendían culto a San Eloy, pero la cofradía no aparece tampoco en la documentación de las parroquias conquenses, ni es mencionada en los documentos personales de los plateros ${ }^{8}$.

Los gremios, en general, funcionaron muy bien allí donde existían y garantizaban la independencia de las asociaciones. Pero en el s. XVIII, el centralismo de los Borbones y su deseo de controlar todas las actividades fue minando esta independencia a través de varias leyes, alguna de las cuales será citada en párrafos posteriores, hasta desembocar en el Real Decreto de Ordenanzas aprobado por Su Magestad a consulta de la Junta General de Comercio y Moneda para todas las platerías de estos reinos y particulares para el Colegio de San Eloy de Madrid, decretada por Carlos III el 10 de marzo de 1771.

Estas ordenanzas derogaban todas las anteriores y constaban de tres apartados de obligado cumplimiento para toda España que incluían la ley de la plata, ventas, visitas etc. El cuarto apartado afectaba sólo a Madrid, ya que trataba sobre el funcionamiento del Colegio por lo que cada uno de los de otras ciudades podían adscribirse a él o seguir con sus propias normas. Las reacciones a este imperativo fueron muy variadas. Valencia, Cádiz, Sevilla y Madrid ofrecieron una fuerte resistencia, mientras Valladolid la acogía docilmente.

En cuanto a la reacción de los plateros de Cuenca, desconocemos cual fue la inmediata a la recepción de la noticia, pero no parece que les afectase demasiado, pues, por la documentación conservada, parece que no tuvo ninguna repercusión. Esto puede deducirse del texto de una carta que el Intendente dirigió al Corregidor, presentada en la Junta del Concejo el 9 de agosto de 1773. En ella insta al Ayuntamiento a hacer cumplir las nuevas ordenanzas ya que había observado que no se estaba haciendo. Los regidores no parecen alterarse demasiado y, como en tantos otros asuntos del momento, formaron una comisión con los capitulares Juan Nicolás de Toledo y Manuel Cardería para que, estudiadas las ordenanzas, redacten un informe sobre el estado de la cuestión, para poder pasarlo al Intendente.

Este asunto no se va a resolver inmediatamente, sino que se va a prolongar varios años, cosa que ocurre en el Ayuntamiento de Cuenca en muchos otros asuntos para los que se nombran comisiones. Casi un año después, el 27 de junio de 1774, se presenta una nueva carta del Intendente instando a los comisarios para que lean las ordenanzas que él mismo les había dado y hagan el informe lo antes posible. Por fin Cardeña y Francisco Antelo, en sustitución de Toledo, presentan el informe el 19 de agosto, aunque no figura su contenido en las actas. Una vez más se demora el asunto pues deciden que pase al Diputado del Común para que emita su propia opinión

La última noticia es confusa e incompleta, pero, si se interpretase al pie de la letra, supondría que los plateros de Cuenca escribieron en estos años las primeras ordenanzas de su historia, para completar el cuarto apartado de la ley de 1771. La noticia es la siguiente. Al hilo de lo anteriormente expuesto en el que vemos al Intendente tratando de hacer cumplir las nuevas normas, el 9 de agosto de 1776 se dice que «... Habiéndose traído las ordenanzas formadas

${ }^{8}$ A. López-Yarto: La orfebrería del siglo XVI ..., pp. 17-18.

$A E A$, LXXVII, 2004, 306, pp. 171 a 201 
por los plateros de esta ciudad...» remitidas por el Intendente, se ordena que pasen a los Diputados y Personero para que emitan un informe. No volvemos a saber nada más del tema y, desgraciadamente, no he conseguido localizar las ordenanzas citadas ${ }^{9}$.

Otro asunto que vincula los plateros al ayuntamiento, es el que hace referencia al nombramiento de Contraste y Marcador. El valor intrínseco de la plata manejada por los plateros hizo que su utilización estuviese muy controlada. Durante varios siglos se siguieron las leyes emitidas por Juan II de Castilla y los Reyes Católicos en lo tocante a la ley de la plata y las que hacían referencia a las personas que debían hacerlas cumplir. Eran estos los Contrastes y Marcadores que debían ser elegidos por los ayuntamientos anualmente. En el s. XvIII los Borbones emiten una serie de nuevas leyes para unificar los criterios seguidos en las diferentes platerías. Para lo que aquí se va a tratar nos interesa tener en cuenta las tres siguientes: El 15 de noviembre de 1730 Felipe V ordena que los Marcadores de cada ciudad se examinen ante el Ensayador Mayor del Reino y, una vez expedido el título, ha de ser presentado ante la Junta de Comercio y Moneda para que esta les entregue el permiso para ejercer el cargo. El 6 de diciembre de 1752 Fernando VI da una nueva ley para que los cargos de Marcador y Contraste recaigan en una sola persona, cosa que ya se venía haciendo desde hacía tiempo en bastantes ciudades. Añade que ejercerían el cargo durante seis años, reelegibles por otros seis. Finalmente Carlos III en sus ordenanzas de 1771 en el título primero capítulo noveno dice: «Todo el oro y plata ... que adquieran los artífices... los han de fundir y reducir a barras o rieles, arreglándolos a la ley correspondiente a ellas... pasaran los rieles a los marcadores públicos, para que reconociéndolos, en pasando de dos marcos por medio del ensaye, o por el toque, o parangón...» ${ }^{10}$. Estas normas se cumplieron con desigual precisión, sobre todo lo que hace referencia al número de años que ejercía su cargo el Marcador.

En Cuenca, en el s. XVI se cumplieron las leyes con gran exactitud durante casi todos los años. Pero en el xVIII el cargo de Marcador debía de ser casi vitalicio. Al menos esto parece deducirse del hecho de que, después de muchos años sin que se haga una cita alusiva al tema en las actas capitulares, el 18 de septiembre de 1783 Tomás López de Mendoza presenta un memorial en el que pide que, por ser ya muy mayor, le exoneren de su cargo de Contraste y Marcador. Le aceptan la renuncia y le piden que devuelva el marco y el material que tenga para poderlos entregar al que le sucediere en el cargo.

Dos meses después se elige a Juan Serrano Briones que había competido para conseguirlo con Ignacio Andrés López. Jura el cargo el día 19 y le entregan el marco oficial. De momento lo tendrá solamente durante un año ${ }^{11}$.

Este nombramiento va a traer algunas complicaciones al no haber pasado Serrano el examen pertinente ante el Ensayador Mayor, y al no pensar el Ayuntamiento en exigirle que lo cumpliera. Esto nos indica que en Cuenca no se tomaban muy en serio la legislación en vigor.

Pero las cosas no quedaron así. El 7 de agosto de 1785 el Corregidor presenta un escrito de Manuel Ximénez, de la Junta de Comercio y Moneda de Madrid, en el que recuerda que el título primero de las Ordenanzas de 1771, dispone que los rieles de plata u oro que pasen de dos marcos deben ser reconocidos por marcadores y que lo ejecuten mediante ensayo, por lo que, al ser una técnica tan delicada hay que comprobar que los que lo hagan sean ensayadores hábiles en ella, y sólo se demostraba si habían sido examinados previamente, o que se hayan comprometido a hacerlo en un plazo máximo de seis meses. Con este examen el Marcador

\footnotetext{
${ }^{9}$ AMC. Libro 343. Libro de acuerdos capitulares, 1773-1774 (doble paginación), fols 66; 48v y 67. Libro 344. Libro de acuerdos capitulares, $1775-1776$, fol. 50.

${ }^{10}$ Parte de estas leyes en la Novísima recopilación de las leyes de España. Mandadas hacer por Carlos IV, Madrid, 1805. Las Ordenanzas de 1771 se publicaron ese mismo año (sin lugar, y sin imprenta).

${ }^{11}$ AMC. Libro 347. Libro de acuerdos capitulares, 1782-1783, fols. 96v, 97v, 101v.

AEA, LXXVII, 2004, 306, pp. 171 a 201
} 
podrá ensayar no sólo los rieles de plata, sino las piezas de más o menos peso que le lleven los plateros. Insiste en que el Ayuntamiento debe hacer cumplir a rajatabla ambas leyes para evitar los perjuicios que se ocasionan a los clientes por los abusos cometidos al no cumplirlas.

Junto a esta carta llega otra firmada por el mismo Ximénez en la que se notifica el envío, de acuerdo con la Junta General de Comercio y Moneda, de un ejemplar del Nuevo arte de Ensayar Oro y Plata, escrito en francés por «el sabio» Mr. Sage. Este es Balthasar-Georges Sage, que era catedrático de docimasia en París, es decir del arte de ensayar los minerales, cuyo libro, de título larguísimo que Ximénez abrevia, fue traducido al español por Casimiro Gómez Ortega y publicado en España ese mismo año de 1785. Este libro debía ser custodiado por el Ayuntamiento y entregado oportunamente al Contraste-Marcador en el momento de su confirmación en el cargo, para que esté al tanto de todas las novedades que aporta al antiguo arte del ensayo.

La Junta del Concejo acuerda hablar con el platero Juan Serrano Briones que había sido reelegido al cabo del año de su primer nombramiento. De esta manera le entregan el citado libro de Sage y le advierten de que, en el término de seis meses, debe presentar al Ayuntamiento el título expedido por la Junta de Comercio y Moneda tras el preceptivo examen, tal y como habían sido requeridos para cumplir las leyes ${ }^{12}$.

Casi un año después, el 29 de mayo de 1786 se presenta un memorial firmado por los plateros Ignacio Andrés López, Manuel y Julián Casado, en el que solicitan se anule el nombramiento de Contraste-Marcador a Juan Serrano Briones, pues se había cumplido con creces el año de su reelección que tuvo lugar en septiembre de 1784, por lo que finalizaba en el mismo mes de 1785. Deciden estudiar todo el expediente para deliberar sobre cual era la decisión que debían tomar. Aunque no figura en los libros de actas, es muy posible que se pusieran al habla con el platero y le recordasen que no había cumplido con su obligación de pasar el examen de Ensayador, pues el 23 de junio de 1786 Juan Serrano presenta un memorial en el que se justifica diciendo que nunca había sido obligatorio ser Ensayador para actuar como Contraste o Marcador en Cuenca, pero que, además, no puede costearse el viaje y estancia en Madrid, ni los derechos del examen. Pide que se le prorrogue otros seis meses el cargo en los cuales intentará reunir el dinero necesario para ir a la Corte. Pero los regidores deciden que ya ha tenido un plazo suficiente desde agosto de 1785 , por lo que hay que nombrar un nuevo platero para que ejerza dicho cargo. Imagino que conocían a la perfección la situación de los plateros, pues, en vez de nombrar a uno directamente, deciden ponerse en contacto con todos los artífices de la ciudad para saber cual de ellos estaba en condiciones de poder presentarse al examen en un plazo de seis meses. No conocemos la respuesta pues no figura en la documentación consultada, pero en virtud de ella el 30 de junio deciden reelegir a Juan Serrano hasta que la Junta de Comercio decida lo que estime más conveniente.

En la última fecha este platero presenta a la Junta de Comercio un memorial en el que relata las circunstancias de su nombramiento y ruega que le liberen de la obligación de examinarse, pues estaba sobradamente capacitado para efectuar todas las operaciones de ese Arte. Pero la Junta contesta que la ley es de obligado cumplimiento, por lo que no pueden eximirlo de la realización del examen, y que, si no está en condiciones de ir inmediatamente a examinarse, debe dejar el cargo. Asimismo la Junta advierte al Ayuntamiento que se ha excedido en sus atribuciones al nombrar un Marcador sólo por un año en lugar de los seis que señala la ley, así como en no exigir desde el principio que los plateros recién nombrados le presenten el título expedido por la Junta. Por todo ello envía al Ayuntamiento copia de las leyes correspondientes de 1752 y 1771 para que las tengan en cuenta en adelante. Además les advierte de que

${ }^{12}$ AMC. Libro 349. Libro de acuerdos capìtulares, 1785-1786, fols. 98v-99v.

$A E A$, LXXVII, 2004, 306, pp. 171 a 201 
saben que hay personas que, sin ser «del Arte de la Platería», ni estar aprobados, trabajan algunas piezas y limpian otras, y les dicen que no pueden consentirlo. Todo esto es presentado en el consistorio el 23 de febrero de 1787.

Finalmente el día 26 del mismo mes Juan Serrano presenta su dimisión y entrega el marco y el libro de ensayar de Sage. En la misma sesión es nombrado para el cargo el platero Ignacio Andrés López, el cual el 12 de marzo agradece la confianza depositada en él y comunica que a lo largo de la primavera irá a Madrid para pasar el preceptivo examen y que le entreguen el título de Ensayador ${ }^{13}$.

Esta resistencia de los plateros a ir a examinarse a Madrid no ocurre únicamente en Cuenca. Conocemos un caso muy parecido en Valladolid, donde, en 1801 hay una pugna por conseguir el cargo entre tres plateros Roque González Téllez, Gregorio de Miranda e Hipólito Bercial del Valle, pero todos se resisten a desplazarse hasta Madrid para pasar un examen que debía de ser muy riguroso ${ }^{14}$.

No he encontrado nuevos nombramientos en los libros de actas revisados hasta 1819. La única noticia de estos años es que el 3 de junio de 1818 se presenta un escrito del procurador Manuel Camarón, en nombre del peostre administrador del gremio de San Eloy, en el que expone que éste ha nombrado examinador al herrero y cerrajero Julián Girondo, pero que no ha aceptado, por lo que pide se revisen las normativas para actuar en consecuencia ${ }^{15}$. Esto demuestra la existencia de un gremio de San Eloy en Cuenca en estas fechas que encuadraba no sólo a plateros, sino también a los cerrajeros y herreros, es decir «oficiales de martillo» como se decía en la solicitud de 1519 y que no conocían muy bien sus propias ordenanzas ya que tenían que recurrir al archivo del Ayuntamiento.

Finalmente hay que hacer constar que el Ayuntamiento fue cliente de los plateros en algunas ocasiones. En septiembre de 1771 se revisan dos trazas presentadas por el repetidamente citado Juan Serrano de una mazas que le habían encargado para sustituir a las antiguas que estaban en muy malas condiciones. Unas estaban presupuestadas en 2.804 reales, las otras en 4.160. Se nombra al regidor perpetuo para que elija las más convenientes. No sabemos que proyecto fue seleccionado, pero el 16 de marzo de 1772 ya estaban terminadas y los regidores deciden que se pague el trabajo del platero. Desgraciadamente las mazas no se han conservado ${ }^{16}$.

El 18 de julio de 1794 toman la decisión de que se haga un cáliz nuevo para el oratorio del Ayuntamiento, pues el presupuesto dado por Juan Serrano por restaurar el antiguo era demasiado elevado y no merecía la pena ${ }^{17}$.

Por último, en 1809 Jacinto Serrano, hijo de Juan como ya se dijo, recibe un encargo del Ayuntamiento. Se trata de una medalla que la ciudad quiere regalar al canónigo magistral por la oración fúnebre que hizo por los fieles difuntos en la guerra contra los franceses. Hay noticias sobre la comisión encargada de evaluar la obra y pagarla al platero el 30 de octubre y el 25 de noviembre de dicho año ${ }^{18}$.

Amelia López-YaRto Elizalde Departamento H. ${ }^{a}$ del Arte. CSIC

\footnotetext{
${ }^{13}$ AMC. Libro 349. Libro de acuerdos capitulares, 1785-1786, fols. 48v, 62v y 68. Libro 3550, Libro de acuerdos capitulares, 1787 , fols. $19 \mathrm{v}, 22 \mathrm{v}$ y $28 \mathrm{v}$.

${ }^{14}$ C. Heredia: Ob. cit., pp. 351-359.

${ }^{15}$ AMC. Libro 370. Libro de acuerdos capitulares, 1817, 1818, 1819, fol. 37v.

${ }^{16}$ AMC. Libro 242. Libro de acuerdos capitulares, 1771-1772, fols. 67, 67v; 38v.

${ }^{17}$ AMC. Libro 357. Libro de acuerdos capitulares, 1794, fol. 87v.

${ }^{18}$ AHM. Libro 365. Libro de acuerdos capitulares, 1809.1810, fols. 51 y 60.
}

$A E A$, LXXVII, 2004, 306, pp. 171 a 201 